\title{
ESTUDIOS
}

\section{Soberanía alimentaria para la cohesión social}

\author{
Jesús Vicens'
}

Resumen: El hambre en el mundo sigue siendo un problema grave. En 2010 el número de personas desnutridas, según el informe de la FAO, alcanzó la cifra de 925 millones. En este artículo quiero presentar, primero, la necesidad de cambiar el sistema mundial dominante de alimentación, basado en la agricultura intensiva, semillas de alto rendimiento, y uso de fertilizantes. Después de 50 años de implantación ha fracasado social y ambientalmente. Los mercados globales han creado mayores desigualdades y una pobreza más profunda. Por ello, la sostenibilidad es un objetivo humanitario fundamental y un asunto de justicia. Segundo, sostengo que la agricultura ecológica es un sistema que favorece a la gente, practicado por comunidades locales en África y en otros continentes. Voy a presentar algunos ejemplos del Informe del Worldwatch Institute, State of the World 2011. En tercer lugar, voy a relacionar el sistema agrícola industrial con el calentamiento global. La agricultura intensiva es dependiente del petróleo en su producción, extracción, transporte y uso de energía. Podría ser un sector renovable si el sistema de producción alimentaria cambiase a pequeña escala, y para beneficio principal de las poblaciones locales y regionales. La reducción de emisiones sería visible. Otro ámbito de atención, es la agricultura urbana, donde la población de las ciudades podría estar bien nutrida mediante la aplicación de los principios ecológicos. Existen actualmente 800 millones de personas que practican la agricultura urbana en el mundo, y producen entre el 15 y el $20 \%$ de la alimentación mundial. Finalmente, hay estilos de vida que preservan el bien común. La conclusión aquí se basa en comprender que la soberanía alimentaria restituye el bien común.

Palabras clave: Soberanía alimentaria, agricultura ecológica y urbana, calentamiento global, resiliencia comunitaria, cohesión social.

Fecha de recepción: 6 de marzo de 2014 .

Fecha de admisión definitiva: 20 de febrero de 2015 .

1 Universidad de Barcelona. 
The humiliated democracy. The political order in times of appeasement

Abstract: Hunger in the world remains a serious problem. In 2010 the number of undernourished people reached 925 million, according to a FAO report. This paper will present first of all the need to change the world's current food system, based as it is on intensive agriculture, high yield seeds, and a use of fertilizers. It has failed both socially and environmentally following its 50 years of implementation. Global markets have created greater inequalities and deeper poverty. Sustainability is a humanitarian, fundamental goal and a question of justice. Secondly, I argue that ecological agriculture is a system that favours people, practiced by local communities in Africa and on other continents. I will present some successful examples from the State of the World 2011. Worldwatch Institute Report. Thirdly, I will correlate the industrial world's agricultural system with Global Warming. Intensive agriculture is oil dependent, for its use of energy, transport, extraction, factories. This could be a renewable sector if the food production system were changed to become small scale, and for the benefit of local regional populations. The reduction of emissions then would be fairly visible. Another ambit to focus on is urban agriculture - city populations could be well nourished through using ecological principles. Right now 800 million people are engaged in urban agriculture around the world, and produce between 15 to $20 \%$ of the world's food. Finally, there are lifestyles that preserve the common good. The conclusion considered here is based on the understanding that food sovereignty restores this common good.

Key words: Food sovereignty, urban and ecological agriculture, global warming, community resilience, social cohesion.

\section{La démocratie humilié. L'ordre poli- tique en temps d'apaisement}

Résumé: La faim reste un problème grave. En 2010, le nombre de personnes sous-alimentées, selon le rapport de la FAO, se élève à 925 millions. Dans cet article, je tiens à présenter, tout d'abord, la nécessité de changer le système de puissance dominante du monde basée sur l'agriculture intensive, semences à haut rendement ef I'utilisation $d^{\prime}$ engrais. Après 50 ans de mise en œuvre a échoué socialement et écologiquement. Les marchés mondiaux ont créé une plus grande inégalité et de la pauvreté profonde. Par conséquent, la durabilité est un objectifhumanitaire fondamental et une question de justice. Deuxièmement, je soutiens que l'agriculture biologique est un système qui favorise les gens, pratiquée par les communautés locales en Afrique et $d^{\prime}$ autres continents. Je vais vous présenter quelques exemples du rapport de I'Institut Worldwatch, State of the World 2011. Troisièmement, je vais vous raconter la relation entre le système agricole industriel et le réchauffement climatique. L'agriculture intensive est tributaire de la production de pétrole, l'exploitation minière, le transport et a consommation d'énergie. Ça pourrait être une industrie des énergies renouvelables si le système de production alimentaire a changé une petite échelle et principal avantage des populations locales et régionales. La réduction d'émissions serait visible. Un autre domaine d'intérêt est l'agriculture urbaine, où la population des villes pourrait être bien nourri par l'application des principes écologiques. Il ya actuellement 800 millions de personnes qui pratiquent l'agriculture urbaine dans le monde, produisant entre 15 et $20 \%$ de l'offre mondiale. Enfin, il ya des modes de vie qui préservent le bien commun. La conclusion ici est basée sur la compréhension que la souveraineté alimentaire rétablit le bien commun.

Mots-clés: la souveraineté alimentaire, l'agriculture écologique et urbaine, réchauffement de la planète, la résilience des communautés, la cohésion sociale. 


\section{Controversia: agricultura intensiva o sostenible}

El hambre en el mundo persiste después de 50 años de agricultura industrial. Ésta, utiliza mucho suelo fértil, mucha cantidad de agua, que extrae de grandes embalses, que alteran el curso de los ríos, semillas muy reactivas y montañas de fertilizantes, sin permitir que los suelos puedan restituirse. Estas son las bases de la agricultura industrial. Los datos de la FAO (Organización de las Naciones Unidas para la Alimentación y la Agricultura) abren un importante debate. ¿Debe ser la agricultura macro intensiva, con todos los factores ya mencionados, la dirección a seguir, o se debe avanzar hacia un modelo ecológico? ¿¿Deben ser los mercados globales el contexto principal de desarrollo o se debería mirar a las comunidades locales como el sendero adecuado para el mismo? En 2010925 millones de personas en el mundo estaban desnutridas, sin suficientes alimentos para sobrevivir (Halweil y Nierenberg, 2011: 4-5). La agricultura se encuentra atrapada en un gran dilema. Los medios dominantes de producción son dependientes del petróleo en un momento de la historia donde las energías fósiles han alcanzado el pico de su producción (Deffeyes, 2001). La agricultura intensiva está poniendo en peligro la fertilidad de los suelos, con un uso excesivo de agua, en un momento donde su escasez en muchos países del sur les lleva a una situación límite. La biodiversidad está seriamente declinando, a pesar de su enorme importancia tanto para proteger la calidad de la vegetación, como para la supervivencia del planeta, y se valora como la sexta extinción masiva (Normander, 2012: 311-323). Los precios de los alimentos fluctúan en el mercado, disparándose, por la presión creciente del consumo de carne, especialmente en Asia, por presiones en producción de trigo en África, y de biocombustibles en Europa y Norte América (Halweil y Nierenberg, 201 1: 5).

La agricultura industrial y la agricultura ecológica empujan en direcciones opuestas. Por esta razón estamos ante una cuestión política y de paradigma económico (Nomander, 2012: 313). La primera opción expulsa a los seres vivos fuera de sus hábitats, hacia la extinción, beneficiando a una parte de la humanidad, la quinta parte que gobierna la economía global, perteneciendo a la clase consumidora. La segunda opción integra la biodiversidad y atiende a todas las poblaciones humanas, pero implica una renuncia significativa al consumismo, la mayoría del cual es superfluo y destructivo. Requiere llevar a cabo cambios culturales de calado, donde la felicidad no se confunda en tener más objetos materiales. Exige permitir que las comunidades de África, Latino América o Asia produzcan sus alimentos para ellos mismos. Hay una dificultad compleja en este cambio, algo esencial en medio de todo esto, y es reorientar los bienes comunes: bosques, océanos, atmosfera y otros, a favor de todos, reduciendo el egoísmo individual y la cultura consumista. Tanto los recursos naturales como los servicios de los ecosistemas, es 
decir, los beneficios gratuitos que proceden de los árboles, las zonas húmedas, la atmosfera, los océanos, etc., son bienes comunes que deben restituirse de su privatización y protegerse del interés particular.

La agricultura ecológica, cercana a los ecosistemas y a la regeneración de los suelos, está en un proceso que contribuye a rehacer la biodiversidad. Tiene en consideración los ritmos más lentos de la naturaleza a diferencia de la aceleración de las sociedades industriales, y considera la biodiversidad de los cultivos una manera de enriquecer los suelos. Es una forma apropiada para construir sistemas de alimentación para la gente y sus comunidades. La urgencia del hambre en el mundo en desarrollo no genera suficiente confianza en las instituciones públicas y autoridades sobre los métodos de producción sostenible. A pesar de ello, la gente, especialmente las mujeres, saben que es una solución real para aliviar el hambre. Y, donde las autoridades locales y las comunidades participan juntas en estos procesos, como sucede en África y otros lugares de Latino América, mejoran la nutrición y la salud. Por ejemplo, en los países en desarrollo, cultivar alimentos en las ciudades, donde vive la mayoría de la población mundial actualmente, no es un pasatiempo sino una necesidad. Estudios llevados a cabo en ciudades de África muestran que las familias que participan en la agricultura urbana, comen mejor, medido en calorías y proteínas, o en relación al índice de crecimiento infantil (Karanja y Nienga, 2011: 118).

En África Subsahariana es donde más confianza hay en esta cuestión, así como en la producción de alimentos y los ingresos que genera. Un tercio de los habitantes urbanos del este de África, de sus pueblos y ciudades, están inmersos en la agricultura. El número de familias del oeste de África que practican la agricultura urbana varía entre un 50\% en Dakar (Senegal) y un 14\% en Accra (Ghana). En Dar es Salaam (Tanzania) el 60\% de la venta de leche se produce en la misma ciudad (Lee-Smith y Prain, 2006). Cuba es un caso especial ya que depende de la agricultura urbana sin haberlo buscado. El $90 \%$ de los productos consumidos en La Habana son cultivados dentro o cerca de la ciudad. El embargo de Estados Unidos, que se mantiene desde los años 90 , ha desafiado la habilidad de la gente cubana para obtener alimentos. Las autoridades públicas crearon una amplia red local en la que tomó parte la gente para conocer las tierras no cultivadas de su radio de influencia, las semillas y la situación del agua (Halweil y Nierenberg, 2007: 52).

Cuando asistimos a este debate es importante introducir la cuestión de la cohesión social: ¿2Puede la agricultura ecológica, que está más cerca de las comunidades, reforzar también los lazos entre la gente y mejorar su interacción, y capacitar así posibilidades de innovación social? En este proceso, ¿̇podría hacerse más visible 
la contribución particular de las mujeres, quienes han ido acumulando mucho conocimiento en los países en desarrollo a lo largo de los años? Esta cuestión se presenta como una alternativa a las consecuencias de la agricultura industrial al haber fracasado para alimentar a la población.

A lo largo de la orilla del río Gambia, un grupo de mujeres ha tenido éxito en llevar adelante una gestión ambientalmente sostenible en el cultivo de ostras. Ésta, es una fuente de alimentación para 15 comunidades que reúne alrededor de unas 6.000 personas. Decidieron prohibir la recogida de ostras durante un año. Al año siguiente, las ostras eran más grandes y los precios un poco más altos y los comerciantes locales y consumidores estuvieron dispuestos a pagarlo. El colectivo de mujeres logró recuperar y conservar los manglares, creando viveros para potenciar las reservas salvajes. También se abrieron a los restaurantes locales y hoteles de turistas (Halweil y Nierenberg, 2011: 3). El marisco, representan una tercera parte de las proteínas consumidas en el mundo y el $15 \%$ de las calorías de la población mundial, más en los países pobres y en buena parte de África Occidental (2011: 4).

Es un buen ejemplo de sostenibilidad. De una intervención ambiental que incorpora la dimensión temporal. Se pueden obtener mejores ingresos al no forzar los ritmos naturales. Actualmente muchas pesquerías están en peligro por haber excedido su capacidad de renovación (Halweil y Nierenberg, 2008: 80-82). El equilibrio temporal está presente en las discusiones sobre sostenibilidad, ya que el mundo moderno está basado en la aceleración del tiempo (Vicens, 2004: 39-58). Es un punto clave en nuestras economías modernas, donde apenas la temporalidad ha sido considerada una dimensión de la realidad, como sí lo ha sido la materia, al ser, ésta última, una realidad más tangible. Los ritmos naturales, la parte más biológica de la dimensión temporal, se han percibido como secundarios, poniendo la fertilidad de los suelos y los ecosistemas en riesgo de perder su capacidad de crear y enriquecer la vida (Adam, 1998: 23-59). Los movimientos sociales y ecológicos, que se presentan como alternativas a las sociedades modernas aceleradas, dan importancia a la dimensión del tiempo. Los conocidos movimientos lentos consideran la producción de alimentos, el cocinarlos y comerlos, como interacciones sociales. Se trata de una relación entre el tiempo y la gente donde el actor principal es la interacción social misma (Milano, 2011: 86).

Los autores Halweil y Nierenberg visitaron en 2009 y 2010, 25 países del África Subsahariana, donde hay un gran problema de hambre, con $1 / 3$ de la población desnutrida. Los investigadores quisieron tomar notas y reunir historias de esperanza y éxito en agricultura que les explicaba la gente local. En Malawi, por ejemplo, 
vieron técnicas de mejora de la eficiencia, usadas por más de 120.000 agricultores, plantando árboles que fijaban el nitrógeno para su posterior cultivo de maíz, multiplicando por cuatro la eficiencia, sin utilizar ningún tipo de fertilizantes. Querían presentar comunidades, países y compañías como referencias de caminos hacia un futuro sostenible (Halweil y Nierenberg, 2011: 6). Es necesario cambiar el sistema de alimentación mundial para cambiar la dirección industrial de la agricultura a una más sostenible y reforzar los principios ecológicos que pueden restituir el equilibrio y la vitalidad de los ecosistemas mediante la biodiversidad. Cuando desaparecen estos principios, las bases de la producción de alimentos se reduce y la seguridad alimentaria para las generaciones futuras está en riesgo. La sostenibilidad es una cuestión central de justicia. Donde el suelo, el agua y la biodiversidad se pierden o se reduce, significa que las bases de la seguridad están en peligro.

El continente africano es cada vez más un banco de pruebas, rico y diverso en materia de agricultura. Por ejemplo, hay una cooperativa de huertos de tejado que suministra alimentos a la ciudad de Dakar (Senegal), que podría servir como ejemplo para orientar a los barrios pobres en Nueva York, zonas urbanas deprimidas. Es un ejemplo de cómo transferir buenas iniciativas de países del sur a países del norte, con situaciones comparables de escasez de alimentos (2011: 7). Pequeños agricultores en todo el mundo pueden hacer mucho para solventar el hambre, mitigar el calentamiento global, y reducir el uso de agua en la agricultura y hacer que haya más agua disponible. Sus innovaciones y experimentos, cuando son compartidas por las comunidades, pueden aplicarse a gran escala y llevar alimentos a la mesa de millones de personas y con ello cambiar el sistema de alimentación mundial. La agricultura engloba una gran parte del planeta. Haciéndola saludable, significa una gran contribución a la sostenibilidad global. Los pequeños agricultores tienen un rol importante en esta contribución, reduciendo los fertilizantes químicos e introduciendo los orgánicos que nutre los huertos, pueden retener más agua de lluvia. Es una forma de cultivo que mantiene los suelos en mejores condiciones y asegura suficiente alimentación. Ello supone también cambios en nuestra dieta consumiendo menos carne, cambios en los métodos de cultivo en favor de aquellos más sostenibles, y cambios en los tipos de alimentos para que los locales estén disponibles para la gente del lugar.

\section{Ecoagricultura: un sistema para la gente y las comunidades}

La agricultura ecológica va de la mano de los ecosistemas. Es una manera de utilizar la tierra para la producción de alimentos sin degradar su entorno. La agricultura se desarrolla para beneficio de los seres humanos, mientras que los 
ecosistemas trabajan para beneficiar al conjunto de los seres vivos. Desarrollar comunidades sin destruir la tierra donde habitan implica visiones del todo: de los humanos y de los seres vivos, un camino de mutuo respeto y beneficio (Canadell y Vicens, 2004: 77-99). Las técnicas suaves de cultivo, que utiliza, o ha utilizado tradicionalmente, la cultura propia de la alimentación de cada región, así como, el conocimiento acerca de los ritmos de cada lugar, del cambio orgánico de las estaciones del año y de las características naturales específicas, se adecuan a esta orientación más sostenible.

Las sociedades modernas, sin embargo, basadas en la producción industrial, la temporalidad lineal, y centrada en unas élites, han abierto demasiados frentes contra sí mismas: hambre, pobreza, desigualdades entre millones de personas, cambio climático, escasez de recursos naturales y de agua, y un planeta donde los suelos, el aire y el agua misma, están contaminadas en una gran extensión, y que produce un volumen de residuos que no llega a absorber. Uno de los principales frentes bajo amenaza es la agricultura. Repensar la producción agrícola cercana a los ecosistemas es colocarse en una perspectiva en favor de la regeneración. Es una posición ética para la humanidad y las futuras generaciones. Algo que no puede eludirse, como dio a conocer el informe Brundtland en 1987 de las Naciones Unidas (UN). Naturaleza y cultura han estado enfrentadas durante siglos de acuerdo a la cosmología dual de Occidente que consideraba que la segunda debía someter y dominar a la primera. En consecuencia la crisis ecológica actual que grapa al conjunto del planeta, empuja hacia la necesidad de formular una nueva cosmología, donde una visión no dual debe ser el referente central (Canadell 2005; 2007: 73-90) y devolver el acople entre la cultura y las culturas con la tierra y los ecosistemas.

Para obtener suelos saludables se requiere recuperar los bosques, los ríos amenazados, las zonas húmedas, los manglares, y otros sistemas naturales, donde pueda haber una biodiversidad importante. En África y en otras partes del mundo podemos encontrar sistemas agrícolas adaptados a los entornos del lugar, los cuales son respetuosos con el medio ambiente. Tienen actividades innovadoras, generalmente accesibles en diferentes países. Louise E. Buck, de la universidad de Cornell, quien es también director del Program Partners Ecoagriculture Landscape y Sara J. Scherr presidenta y consejera del Partners Ecoagriculture (2011) sitúan la necesidad de recuperar los métodos rurales de supervivencia como un aspecto importante y motivador de estas actividades. Así como, recuperar las formas de dinámica social de las comunidades que han implicado y valorado otras funciones en la agricultura. La producción no es la única función. Hay, por ejemplo, una preocupación creciente por los suelos y los sistemas de agua degradados: ríos, 
lagos, acuíferos, como resultado de prácticas agrícolas que han sido corrientes pero que rompe estos sistemas y los contamina. Prácticas procedentes de la agricultura intensiva: mucha agua, semillas de alta reacción y muchos fertilizantes artificiales. Ello ha provocado que muchas plantas silvestres y especies animales estén amenazadas. La expansión de las tierras de cultivo hacia los bosques, sabanas, y zonas húmedas, así como los métodos usados que incrementan los daños a los ecosistemas, son causantes de estas amenazas y pérdidas de sus hábitats.

Sin embargo, un giro hacia la agricultura ecológica hace que cada vez más haya una mirada a los agricultores como actores capaces de mitigar el cambio climático por su habilidad en absorber dióxido de carbono en las plantas y los suelos, como resultado de generar paisajes ecoagrícolas diversos. Hay un interés en observar y confirmar si los agricultores son capaces de cambiar los sistemas mundiales de producción intensiva a ecológica. Es un aspecto político entre los bienes comunes o el beneficio privado y minoritario de capital financiero y las agroindustrias, que consideran la alimentación una mercancía objeto de negocios.

Para alcanzar un paisaje de agricultura ecológica, el cual implica producción agrícola, desarrollo rural y gestión de los ecosistemas, los autores mencionan dos estrategias necesarias: una, introducir prácticas de producción ecológica en las explotaciones agrícolas existentes en lugar de seguir la producción intensiva; la segunda es articular una colaboración entre todas las partes interesadas en acción para tratar con los recursos naturales y agrícolas en una forma integrada de la tierra (Buck y Scherr, 2011: 17-18). Las prácticas ecológicas y la participación entre estos actores están en manos de la gente y pueden llevarlas a cabo. Las repercusiones de cada una van más allá de situaciones específicas de cualquier comunidad o ecosistema. Hay una trascendencia implícita, más allá de resoluciones específicas. Es importante ser conscientes de esto como un elemento de la nueva cosmología no dual (Canadell, 2005).

Un objetivo de la agricultura ecológica es incrementar la riqueza económica de los suelos, así como del agua y de la energía, y satisfacer las necesidades de las familias y las comunidades, mientras se suministran también productos, que se hallan en los mercados de la agricultura intensiva, con una calidad de origen para vender a escala local y mercados regionales. Un aspecto importante de este enfoque es la diversidad de los sistemas agrícolas en una variedad de combinaciones de cultivos. Árboles, horticultura, apicultura, y otras formas de diversificación agrícola. Para alcanzar paisajes ecoagrícolas, que favorecen la soberanía y seguridad alimentaria, y recuperan sistemas agua, o preservan la biodiversidad, un buen objetivo es implicar a las organizaciones de agricultores y las comunida- 
des agrícolas a colaborar con los grupos responsables de gestionar los bosques, - cualquier otro ecosistema. Así como practicar la gestión adaptativa, esto es, ajustar los planes cuando fuera necesario para responder a cualquier imprevisión, situaciones cambiantes, y aprovechar los conocimientos comunitarios adquiridos (Buck y Scherr, 2011: 20-21).

La agricultura, como otras esferas de la vida cotidiana: vivienda, salud, cultura, se ha puesto en el mercado para hacer negocios lucrativos en base a la cultura del consumo como su factor principal. Estos sectores de la sociedad mencionados son sistemas sociales básicos que generan interacción entre la gente. Por ello, el valor que se deriva de la experiencia de localidad hace que estas relaciones articulen un sentido de la vida y un estilo cultural propio de cada comunidad. No reduce la visión y percepción cosmopolita de la vida, sino que evita el reduccionismo de un solo estilo de vida, el consumismo de los países ricos, que ha puesto en estado de alerta ecológico al planeta y de riesgo de fisura social a la humanidad. Son valores más allá del lucro financiero generado en los mercados globales. Si la cultura de la localidad ocurre en países en desarrollo, donde hay un elevado nivel de necesidades básicas no cubiertas, como en África, es fácil encontrar innovaciones emergentes que buscan maneras de satisfacer las necesidades básicas, como la nutrición. La agricultura está muy enraizada en la gente y es un marco real para hacer posible estas iniciativas.

Tres ejemplos para ilustrarlo. Primero, en Tapajos, en el estado brasileño de Pará donde los agricultores indígenas han creado cultivos de árboles cercanos a las zonas protegidas de bosque en la periferia de las áreas de producción intensiva. Haciendo esto han reducido la conversión de hábitats naturales en producción industrial, mientras mejoran el valor del hábitat de estas áreas que ya están cultivadas, contribuyendo a crear una matriz benigna para los hábitats fragmentados. Segundo, en Zimbabue hay un plan estratégico del paisaje con una zona de 8.000 hectáreas de pasto colectivo en Dimbangombe que proporciona un hábitat importante para la fauna y flora dentro del parque nacional de Hwange y Zambezi. Una gestión previa de pastos pobres ha significado el deterioro en la calidad de la hierba, del suministro de agua y de la biodiversidad que mantiene la agricultura, la flora, la fauna y el turismo local. Los métodos de supervivencia estaban en peligro y aquellas personas a cargo de la tierra introdujeron rotación de pastos con un propósito innovador. Tercero, iniciativas en Kericho, Kenia, han capacitado a los miembros de la cooperativa local de té y a pequeños agricultores a gestionar 8.000 hectáreas de plantación usando Principios de la Plataforma de Iniciativas de Agricultura Sostenible. Los agricultores usaron cubiertas vegetales y cultivos entre las plantaciones de té, para incrementar los niveles de materia orgánica, mientras 
el terraplén, el uso de agua en medidas micro y el drenaje preservan y enriquecen tanto el suelo como el agua. No utilizan insecticidas, ni fungicidas y hay terrenos de bosques, zonas húmedas, paravientos y barreras hechas en los márgenes de las tierras (Buck y Scherr, 2011: 21-22). Cada uno de estos ejemplos, y muchos otros, generan no solamente protección ambiental, sino también cohesión social. La protección ecológica va acompañada de la seguridad comunitaria, del empoderamiento de la gente que ve que sus tierras y sus comunidades se desarrollan en una matriz interdependiente de bienestar.

No obstante, emergen dos cuestiones claves de la controversia entre la agricultura intensiva y la agricultura ecológica que la investigación deberá seguir en la observación de los casos y el análisis de los experimentos. ¿Qué cambios deben hacer los sistemas de agricultura ecológica, en los paisajes ecoagrícolas, para satisfacer la demanda de alimentos cuando ésta crece tan rápidamente? ¿Pueden competir económicamente con la agricultura del monocultivo, con sus semillas de alta reacción y recursos agroquímicos? Los paisajes ecoagrícolas están pensados para satisfacer objetivos ecológicos, sociales y económicos, y deben ser evaluados en relación con estos múltiples criterios de eficiencia que son las tres dimensiones de la sostenibilidad: medioambiente, sociedad y prosperidad.

Las prácticas de la agricultura ecológica están promovidas, como señalan Buck y Scherr, cuando la inseguridad alimentaria es alta, y la presión para intensificar la agricultura es igualmente alta. También se promueven cuando el deterioro de los suelos es una barrera a la intensificación de la agricultura y cuando los ecosistemas deteriorados amenazan la producción de alimentos y la sostenibilidad. Todo esto sucede cuando hay una reducción en los flujos de agua o en la calidad de la misma para irrigación, como resultado de inundaciones o de la pérdida de recursos de pastoreo (Buck y Scherr, 2011 : 24). En los países en desarrollo en particular, el movimiento de soberanía alimentaria ha reunido juntos a pequeños agricultores, trabajadores agrícolas, pescadores, pastores y artesanos, para devolver el control de la producción y el consumo a los sistemas de alimentación local (2011: 25).

\section{La agricultura frente al cambio climático}

El papel de los agricultores y de la agricultura en mitigar los efectos del cambio climático puede ser relevante. El sistema agrícola mundial es dependiente del petróleo, como lo son otros sectores de la economía: la energía, el transporte, las industrias extractivas, entre otros. La economía entera se fundamenta sobre las 
fuentes fósiles de energía. La sostenibilidad, en cambio, se orienta en dirección a la descarbonización de sus fuentes de energía. Los métodos de la agricultura sostenible, con una producción a pequeña escala y para beneficio de la población local y regional pueden ser ejemplos visibles de reducción de emisiones de carbono. Un objetivo es contribuir a un cambio en este sector económico primario muy sensible; de ser causa del calentamiento global, debido a su sistema industrial intensivo, puede pasar a ser reparador. Los agricultores pueden liderar con ello la seguridad para nutrir de alimentos a su gente y comunidades y a la vez mitigar las consecuencias negativas del cambio climático para su gente. Ciertamente los ejemplos tienen todavía poca relevancia cuantitativa, aunque son una referencia significativa donde mirarse aquellas comunidades que quieran optar por seguir la senda de la sostenibilidad de los cultivos.

Una de las principales políticas para reducir el calentamiento global ha sido invertir en plantación de árboles que aumentan las áreas de bosque y frenan la erosión del suelo. Sin embargo, aunque esta política se ha llevado a cabo como una estrategia para equilibrar los problemas que se han dado de calentamiento global relacionado con la agricultura (Brown, 2002: 166-182), hay un sentido general de que no necesariamente ha podido ser una política adecuada ya que ignora los resultados que se dan a gran escala. Por ejemplo, no tener en cuenta la matriz natural de las plantas locales y no reconocer las necesidades de un lugar específico. Puede ser una política errónea, que utiliza mucho capital con pocos resultados. El clima en África y en muchos otros lugares del mundo será diferente en las próximas décadas (Stern, 2007; Lobell y Burke, 2011:90). Poner la mirada en la investigación y experimentación que los agricultores locales intentan implementar puede ser una mejor estrategia. Los pequeños agricultores de África y aquellos de otros lugares están llevando a cabo constantemente investigaciones. Ellos mismos hacen sus propias investigaciones en sus tierras, árboles y animales. Buscan soluciones cuando encaran los retos que tienen delante. Buscan las técnicas más apropiadas en función de su propio entorno local (Letty, Noordin, Magagi y Waters-Bayer, 2011: 55).

El cambio de paradigma para incrementar la superficie boscosa debe ser la regeneración ecológica, gestionada por los agricultores locales desde perspectivas naturales. África está en la vanguardia de estas iniciativas. Chris Reii (2011) especialista en gestión sostenible de los suelos en el Centro de Cooperación Internacional en la Universidad Libre de Ámsterdam y un facilitador de las Iniciativas de Reverdecimiento de África (Africa Re-greening Initiatives) nos informa de los agricultores de la región de Maradi y Zinder, en el sur de Níger. Han protegido y gestionado la regeneración espontánea de algunas especies forestales en sus 
campos en una superficie de 5 millones de hectáreas. Es un ejemplo de una actividad a gran escala en uno de los países más poblados de la región. Ha producido la transformación ambiental más grande del Sahel, y una de las más grandes de África (Reii, 201 1: 92-93). Los beneficios ambientales y sociales de estas iniciativas son significativos para asegurar la viabilidad de los proyectos que intentan regenerar la tierra deforestada. El autor menciona como beneficios ambientales la reducción de las temperaturas y de la velocidad de los vientos que provocan los árboles y evitan la evaporación, mientras retienen el carbono e incrementan la biodiversidad. Algunas de las especies de árboles fijan el nitrógeno y mejoran los suelos. Y, como beneficios sociales están la seguridad alimentaria para las familias, en comparación con los sistemas supuestamente más productivos; mejor resistencia en los periodos de sequedad, y un nivel más complejo que reduce el hambre, la pobreza y la mortalidad infantil. Reduce a su vez el tiempo de las mujeres para recolectar leña, así como, las disputas entre agricultores y pastores en estas áreas regeneradas (Reii, 2011: 93).

Combinar árboles, plantas y arbustos, con una observación cuidadosa y una gestión de los agricultores y pastores, siguiendo ritmos más naturales, lleva al conjunto del sistema del paisaje ecoagrícola a un equilibrio. No erosiona los suelos, como sí hacen las semillas de alta reacción, los fertilizantes y el uso de más agua. También mejora la política de plantar un muralla de árboles, cuando solamente sobreviven entre el $20-30 \%$ después de dos o tres años. Las políticas ecológicas, sin embargo, protegen la tierra, sus comunidades, y mitigan las consecuencias del calentamiento global. Debemos reflexionar si las grandes estrategias, como la agricultura intensiva o la reforestación intensiva, funcionan bien. El conocimiento de las experiencias locales y naturales y el rol central que juega la gente que gestiona y trabaja la tierra son más significativos cuando se quieren explorar alternativas al sistema de alimentación industrial. Los impactos producidos por la agricultura intensiva han alcanzado un límite en las posibilidades para la supervivencia. Éstas posibilidades abarcan los umbrales de sostenibilidad en términos sociales: hambre, nutrición, seguridad y soberanía, o en términos ambientales: fertilidad, biodiversidad, agua y alimentos.

Estos ejemplos de los agricultores protegiendoy gestionando la regeneración espontanea de especies forestales en las explotaciones del Sahel, condujo a la Iniciativa de Reverdecer el Sahel en 2007 (Sahel Re-greening Initiative). Había un programa instigado inicialmente en Burkina Faso y Mali en 2009 para incrementar los éxitos, que más tarde se extendieron a Níger y Etiopía, con la decisión de ampliar el programa de los que hoy se conoce como Iniciativas de Reverdecimiento de África (ARI, en inglés). Este proyecto ha identificado diferentes mecanismos acerca de 
aquellas actividades que tienen por objetivo transformar la desertización y deterioro de la tierra. Por ejemplo, seleccionando aquellas organizaciones en cada país con experiencia de participación en la gestión de recursos naturales, donde la gente es la razón de ser principal. Otro ejemplo es la identificación y análisis de casos exitosos de regeneración ecológica. Otro son las visitas de las autoridades locales electas y regionales, y técnicos y expertos, a los lugares donde los agricultores han regenerado sus tierras. El cuarto sería ayudar a la gente a aprender de otros agricultores. Un quinto mecanismo podría ser el establecimiento de instituciones municipales, para la tarea de gestión de los árboles, donde probablemente los aspectos técnicos del área sean sencillos y donde los factores de capital social son más complejos. Otro sería la incorporación de la regeneración ecológica en los proyectos más amplios de desarrollo agrícola nacional. Atender a los medios de comunicación local, nacional o internacional para mostrar la transformación de los sistemas de producción a través de películas es otro mecanismo. Y otro, sería buscar compromisos a largo plazo para la regeneración ecológica de todas las partes interesadas. Finalmente, el desarrollo de las actividades de investigación en la regeneración ecológica (Reii, 2011: 93-98).

Otro problema significativo del cambio climático se deriva de la industria de la alimentación y concierne a los tipos de dieta. Aquí la industria agrícola es una de las causas del cambio climático. La agricultura en los países en desarrollo puede ser una de las fuentes principales de emisiones de gases de efecto invernadero, aún sin industria pesada, debido a la participación específica en la deforestación. Anna Lappé (2011), cofundadora de la Fundación Planeta Pequeño y autora de Diet for a Hot Planet: The Climate Crises at the End of Your Fork and What You Can Do About It, provee algunos datos en emisiones de dióxido de carbono como resultado de la pérdida de bosques. Menciona que representa el $17 \%$ de las emisiones antropogénicas y la producción de ganado el 18\%. Las emisiones de gases invernadero derivadas de la comida se producen en cada paso de la cadena alimentaria: el trabajo de la tierra, el procesamiento, empaquetamiento, transporte, comercio, restaurantes, consumo doméstico y residuos. A pesar de ello, los agricultores innovadores en todo el mundo, quienes utilizan los principios de la agricultura ecológica muestran el potencial de las explotaciones sostenibles para alimentar a la gente sin contribuir a la crisis climática. El punto clave es no depender del petróleo. Estas explotaciones ecológicas pueden ayudar al mundo a adaptarse al cambio climático. Los fenómenos meteorológicos extremos ocurrirán con mayor frecuencia. Un sistema de alimentación sin fertilizantes, pesticidas, y que usa técnicas para cubrir los suelos, rotar las cosechas, cultivando con insectos beneficiosos, va creando un suelo sano (Lappé, 2011: 100-101). Lo que la gente come es esencial para contribuir al calentamiento global o mitigar sus efectos 
negativos. Aunque es un tema muy sensible, es necesario discutirlo, debido a los cambios en el dietario con los ingresos mayores en los países en desarrollo, crece un mayor número de vacuno.

\section{La agricultura urbana: como alimentar las ciudades}

Podemos ver que hay un contrate drástico entre gente rica y pobre en las ciudades de los países en desarrollo. Hay viviendas en las áreas ricas de estas ciudades que lo tienen todo: jardines, infraestructuras, servicios, confort y seguridad. Muy cerca de ellas hay suburbios ("slums"): lugares donde las viviendas son muy precarias, sólo barracas, sin servicios, sin comida, ni suministro de agua o energía, sin acceso o conexión con el resto de la ciudad (UN, Global Report on Human Settlements, 2003). La gente en los "slums" es muy vulnerable al crimen, a la inseguridad alimentaria, a la pobreza y malnutrición. Todos estos factores convergen juntos. Comprar comida, por ejemplo, puede representar el $80 \%$ del dinero de estos hogares pobres, dicen Nancy Karanja y Mary Nienga (2011), investigadoras del centro Urban Harvest, con sede en Nairobi, (Kenia). La gente urbana pobre está hambrienta y en una situación de desventaja en comparación con los pobres rurales. Estas situaciones son extremadamente desafiantes, aunque se han encontrado algunas soluciones innovadoras que prometen ser una ayuda real, como cultivar alimentos para el consumo propio y para venderlos en los entornos urbanos. En el África Subsahariana y en el mundo desarrollado diferentes comunidades han creado sistemas de agricultura urbana adaptados a lugares específicos, que remiten a factores nutricionales críticos, igualdad de género, ingresos y seguridad alimentaria. Las autoras proveen una tabla con innovaciones para nutrir las ciudades. Por ejemplo, huertos verticales para cosecha urbana en Kibera, Nairobi (Kenia); Pequeños huertos en África Subsahariana; huertos en los tejados, como en Eagle Street, New York; comunidades agrícolas en Sudáfrica (Katanja y Nienga, 2011: 118-119). Algunas ciudades tienen una larga historia en seguridad alimentaria que reduce el hambre de la gente más vulnerable.

Las autoras mencionan que las encuestas de la segunda parte de los 90, en 24 ciudades de África y Asia, muestran que la gente de los hogares pobre quienes practicaban agricultura urbana tenía mejores dietas y nutrición, y solían comer más. Datos de Kampala (Uganda) en los 90 muestran que los niños de los hogares que practicaban agricultura urbana estaban mejor alimentados en comparación con aquellos hogares que no lo practicaban. Cultivar la propia comida hace posible ahorrar dinero, y es una fuente de trabajo e ingresos, ambos muy necesarios cuan- 
do se vive en los "slums". Estos ahorros pueden destinarse a la educación de los niños, ropa, y cualquier otra necesidad doméstica (Karanja y Nienga, 201 1: 118).

Cultivar alimentos en las ciudades genera claros beneficios a varios niveles sociales: nutrición segura, creación de comunidad, y mejoras en el estatus de la mujer y del medio ambiente. La agricultura urbana mejora la seguridad alimentaria en muchas ciudades y es un instrumento positivo para los grupos vulnerables. Hay alrededor de 800 millones de personas que practican la agricultura urbana en el mundo, que producen entre el 15-20\% de la alimentación mundial. De estos, 200 millones producen alimentos para los mercados comerciales urbanos y suministran unos 150.000 millones de puestos de trabajo. Las proyecciones para el año 2020 son que entre 35 y 40 millones de africanos viviendo en las ciudades dependerán de la agricultura urbana para cubrir sus necesidades alimentarias. De esta manera podrían obtener el $40 \%$ de sus calorías diarias y el $30 \%$ de sus necesidades proteínicas. (Karanja y Nienga, 2011: 119-120).

Hay algunas ventajas en producir alimentos en las ciudades comparadas con las zonas rurales: la proximidad de los mercados, bajo costes de transporte y una reducción en las pérdidas que siguen a la cosecha, ya que hay menos tiempo entre cosechas (Karanjay Nienga, 201 1: 120). Las mujeres tienen un papel importante en este proceso. Trabajan juntas, aprenden y se enseñan unas a las otras, comparten cualquier información y conocimiento de los mercados o de cualquier plantación que hagan. Los lazos sociales y comunitarios entre ellas son especialmente relevantes, particularmente cuando hay indigencia, donde la esperanza emerge con mayor claridad.

Construir comunidades es otra dimensión que emerge de la agricultura urbana. Es el resultado de la interacción social que crean los jóvenes, las mujeres y los grupos vulnerables de autoayuda. Se encuentran como resultado de estar implicados en la agricultura urbana y tienen la oportunidad de organizar e intercambiar información. Otra manera de construir comunidades en las ciudades son los huertos escolares, y que extienden currículos extraescolares. Los estudiantes adquieren conocimientos prácticos de como plantar, cultivar y cosechar alimentos, aprenden a la vez acerca de las cuestiones sociales relevantes como el liderazgo, la organización social y las responsabilidades. Las escuelas que tienen terrenos, agua y edificios, se han usado como agentes de diseminación de ideas de la agricultura urbana y producen semillas. El ayuntamiento de Kampala, por ejemplo, ha abierto escuelas de extensión para preparar y proveer soporte técnico y material a los agricultores urbanos, mientras muchas escuelas primarias tienen huertos para enseñar sobre agricultura urbana y nutrición a sus estudiantes (Ssekyewa, y otros, 2007: 149-163). 
Las mujeres que hacen lo mejor posible para alimentar a sus familias, a veces en situaciones muy difíciles, son las que además nutren las ciudades y necesitan soporte institucional. La agricultura urbana puede ayudar a mejorar la calidad del entorno. La gente pobre en las zonas urbanas es inteligente y reciclan los residuos para hacer compost. Haciendo esto reducen la polución que las ciudades deben encarar y tratar con los residuos agrícolas e industriales. En Kenia, por ejemplo, los restos orgánicos son usados ampliamente. También reutilizar el agua residual es una práctica extendida en muchos países como China, Egipto, Méjico, Perú, Vietnam, donde ha sido una fuente de nutrientes para cultivar. Al menos 2 millones de hectáreas de tierra en los países en desarrollo son regados usando agua residual (Lee-Smith y Lamba, 1991: 37-40).

Por otro lado, la silvicultura también beneficia el medio ambiente local. Los árboles contribuyen en producir sombra, frutos y frutos secos. Los árboles sirven también como rompe vientos, estabilizan el suelo y disminuyen la erosión, así como mejoran la calidad del aire y del agua que va a los acuíferos. Los árboles en las ciudades son una verdadera ayuda en la disminución de dióxido de carbono, embellecen la zona y preservan la biodiversidad. Los bosques pueden reducir la contaminación del aire y mitigar los efectos de las tormentas y lluvias torrenciales en las ciudades. Todos estos factores combinados conjuntamente contribuyen a la sostenibilidad de los centros urbanos. Los investigadores de las decisiones políticas y los planificadores urbanos han entendido que los bosques urbanos ayudan a mejorar la vida y el trabajo en las ciudades (Karanja y Nienga, 2011: 123-124).

\section{Discusión}

En esta última parte del artículo quiero discutir tres aspectos diferentes. El primero relacionado con la cuestión central del artículo. ¿Puede la agricultura ecológica alimentar el mundo? ¿ंPuede satisfacer la demanda mundial de alimentación? $\mathrm{O}$, en términos macro-estructurales, ¿̇sólo la agricultura intensiva puede hacerlo? El segundo, está relacionado con el poder y la política, y ¿̇a quién pertenece tomar las decisiones que afectan a la nutrición del planeta? ¿ $\dot{i}$ Tal vez caiga del lado de las comunidades y la gente en un nivel micro-económico? $\mathrm{O}$, ¿debe ser una cuestión de estructuras macro-económicas? ¿ ¿Tiene la gente, sus familias y comunidades,

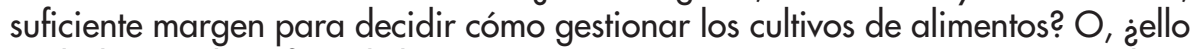
es dado para beneficio de las macro-corporaciones e instituciones macro-gubernamentales? El tercer aspecto, está relacionado con aquellas formas que capacita a la gente a encontrar soluciones para su pobreza y hambre; sus habilidades 
para innovar y buscar soluciones reales haciendo más resilientes a las comunidades y empoderando a su gente. Hay actualmente suficientes ejemplos de buenas organizaciones en el mundo, que muestran que un cambio social importante está emergiendo, cambiando el equilibrio de poder en favor de la gente, y reduciendo los márgenes de ganancias de los mercados globales y las corporaciones. Millones de personas están tratando con soluciones ecológicas en sus economías locales, están trabajando en micro proyectos y encuentran verdaderas alianzas en métodos sostenibles de cultivos, aunque todavía no tenga peso cuantitativo suficiente. El bien común está en el horizonte de la gente pobre que vive en los "slums", donde ven y sienten como propias la pobreza y el hambre.

En relación con el primer punto, quiero añadir una reflexión en la conversación de la controvertida cuestión que aparece en muchos debates sobre alimentación, acerca de la habilidad de la agricultura intensiva para alimentar el planeta como la única vía posible que tenemos delante, al ser una noción imprecisa y confusa. Debemos añadir a estos debates la necesidad de cambiar que tiene la sociedad y el medio ambiente, y que justifica el apoyo a la agricultura ecológica. El sistema de alimentación mundial actualmente está basado en una estructura macroeconómica, dirigida a incrementar constantemente los depósitos de capital, mientras deja de lado los costes sociales y ambientes, sin contabilizarlos.

El sistema social mundial está todavía enraizado en las comunidades, aunque éstas puedan romperse con la globalización, aún están presentes sus raíces y pueden rehacerse fácilmente, como los árboles de un bosque, o los fondos del océano, sí se les deja sin interferir o estorbar. Cuando el sistema social es desestabilizado en cualquier país o región, empiezan a establecerse lazos entre la gente, generando solidaridad, y una tendencia emerge hacia la reconstrucción de la cohesión social. Es una dimensión natural que junta a la gente. Empuja a las comunidades, a la gente, a las familias a intentar y explorar su fortaleza y resiliencia. Podemos encontrarlo hoy en muchos lugares del mundo. Los problemas socio económicos que enfrentamos actualmente son demasiado grandes para ser entendidos por cualquier persona. En cualquier campo dado, alimentación, energía, movilidad, agua, y muchos otros, la misma estructura de estos problemas es muy grande, complicada y confusa. La implementación de los mercados globales como norma principal del comercio ha establecido la mentira y el egoísmo como norma. Las señales que se envían al conjunto de la sociedad están lejos de ser verdad. Contrariamente, están hablando de una ficción, lejos de la realidad. Los problemas ambientales, especialmente el cambio climático, son tan intensos, que cualquier proyecto social para rescatar la situación es impredecible. 
Cualquier experimento con suficiente capacidad de motivar a la gente y que devenga en un movimiento social es una referencia a considerar seriamente. Sea en las zonas rurales o en los barrios urbanos, los proyectos de agricultura ecológica son todavía pequeños experimentos, pero son rutas claras para liderar una salida al colapso en cualquier lugar del mundo, para al menos las tres cuartas partes del mundo que no están considerados dentro del diseño de los mercados globales. En este artículo he acercado ejemplos que refieren estas nociones, mostrando la resiliencia de la gente. Han empezado a transferirse estas prácticas y resultados a otros lugares. La federación de pobres urbanos, los suelos compartidos, y los presupuestos participativos, son ejemplos de innovación que llevan a repensar de quien es la ciudad, a quien pertenece y como funciona (Perlman y $\mathrm{O}^{\prime}$ Meara Sheehan, 2007: 181).

\section{Bibliografía}

ADAM, B. (1998) Timescapes of Modernity. London, Routledge.

Brown, L. R. (2002) Ecoeconomia. Barcelona, Centre UNESCO de Catalunya.

BUCK, L.; SCHERR, S. (2011) "L'ecoagricultura com a norma", en L'estat del món 2011. Worldwatch Institute. Barcelona, UNESCOCAT.

CANAdell, À. y Vicens, J. (2010) Habitar la ciudad. Madrid, Miraguano.

- (2004) "Els sistemes vius", en La textura de la vida. Girona, Documenta universitària.

Canadel, À. (2005) La noció de temps en Raimon Panikkar. Barcelona, Universitat de Barcelona. www. tdx.cat/handle/10803/2040

- (2007) "Sostenibilitat. Una cultura de la interdependència". Sostenible?, n 9. Barcelona, Universitat Politècnica de Catalunya, pp. 73-90.

DAVIS, M. (2007) "La ecología de las áreas urbanas hiperdegradadas", en Planeta ciudades miseria. Madrid, Foca.

DefFEYES, K.S. (2001) Hubbert's Peak: The Impeding World Oil Shortatge. Princeton (NJ), Princeton University Press. 
Halweil, B. y Nierenberg, D. (2007) "Farming the Cities", in State Of The World 2007. Worldwatch Institute. Washington, DC, Norton.

- (2008) "Carn i peix: els ingredients amb major cost de la dieta global", en L'estat del món 2011. Worldwatch Institute. Barcelona, UNESCOCAT.

- (2011) "Un nou camí per posar fi a la fam", en L'estat del món 2011. Worldwatch Institute. Barcelona, UNESCOCAT.

Karanja, N. y Nuenga, M. (2011) "Com alimentar les ciutats", en L'estat del món 2011. Worldwatch Institute. Barcelona, UNESCOCAT.

LAPPÉ, A. (2011) "La crisi climàtica que se serveix als nostres plats", en L'estat del món 2011. Worldwatch Institute. Barcelona, UNESCOCAT.

LEE-SMith, D. y PrAIN, G. (2006) Understanding the Links Between Agriculture and Health, Focus 13. Washington, DC: IFPRI.

LEE-SMith, D. y LAMBA, D. (1991) "The Potential of Urban Farming in Africa", Ecodecision, Diciembre 1991

LetTY, B.; Noordin, Q.; MagaGl, S. y Waters-Bayer, A. (2011) "Els agricultors encapçales les activitats de recerca i desenvolupament", en L'estat del món 2011. Worldatch Institute. Barcelona, UNESCOCAT.

LobeLL, D. y BuRKe, M. (2011) "L'enfocament agnostic de l'adaptació al canvi climàtic", en L'estat del món 2011 . Worldwatch Institute. Barcelona, UNESCOCAT.

MILANO, S. (2011) "Protegir la biodiversitat alimentària local", en L'estat del món 2011. Worldwatch Institute. Barcelona, UNESCOCAT.

NORMANDER, B. (2012) "Biodiversidad: combatir la sexta extinction masiva", en La situación del mundo 2012, Worldwatch Institute. Barcelona, Icaria.

Perlman, J. y O'Meara Sheehan, M. (2007) "Fighting Poverty and Environmental Injustice in Cities", in State Of The World 2007. Worldwatch Institute. Washington, DC, Norton.

REIJ, C. (2011) "Inversió en arbres per mitigar el canvi climàtic", en, L'estat del món 2011. Worldwatch Institute. Barcelona, UNESCOCAT. 
SsekYEWA et Aul, (2007) "Vegetable Gardening in PrimarySchools and Its Impact on Community Livelihoods in Uganda" Journal of Sustainable Development in Africa, vol.9, $\mathrm{n}^{\circ} 2$

Stern, N. (2007) "Implicaciones del cambio climático para el desarrollo", en El informe Stern. Barcelona, Paidós.

United Nations Human Settlements Programme. (2003) The Challenge of Slums. London, UN-HABITAT / EARTHSCAN

VICENS, J. (2004) "Tiempo tecnológico y tiempo natural", en J. VICENS (ed.) Tiempo y cambio social. Valencia, Germania. 